\title{
Management of a Visible Nodule Following Poly-L-Lactic Acid Injection in the Periorbital Area
}

\author{
Jin Su Shin, Eun Soo Park, \\ Jin Young Kim \\ Department of Plastic and Reconstructive \\ Surgery, Soonchunhyang University \\ College of Medicine, Bucheon, Korea
}

Poly-L-lactic acid (PLLA) is a synthetic injectable medical agent for use as an effective soft tissue filler to treat facial fat volume loss, although complications including nodule formation do occur. We describe a case of foreign body granuloma formation in an immunocompetent patient who received PLLA in the periorbital region for soft tissue aesthetic enhancement and treated with surgical intervention by subciliary approach.
This work was supported by the Soonchunhyang University Research Fund.

No potential conflict of interest relevant to this article was reported.
Keywords Complications, Dermal fillers, Injections, Lactic acid

\section{INTRODUCTION}

Poly-L-lactic acid (PLLA) is a synthetic injectable medical agent used to treat patients with loss of volume in the face, chest, and hands. It consists of a biodegradable and bioresorbable powder, a synthetic nonpermanent polymer. PLLA was introduced in Europe in 1999 (NewFill; Ashford Aesthetics Inc., Brussels, Belgium) under the category of wrinkle filler [1]. The Food and Drug Administration (FDA) approved it for United States use in 2004 (Sculptra; Dermik Laboratories, Berwyn, PA, USA) for correction of facial fat loss secondary to lipoatrophy in human immunodeficiency virus (HIV) positive patients and in 2009 as a cosmetic volumizer for soft tissue enhancement in the non-HIV-positive population.

PLLA is very effective at increasing tissue volume and it has been found to provide clinical benefits lasting up to 2 years [2]. Although it has been further suggested to have a low likelihood of adverse events [1], one of the most common side effects associated with PLLA is the occurrence of subcutaneous nodules [3]. Nodule formation may be cosmetically disfiguring and frequently leads to a therapeutic challenge.

Received: May 17, 2016 Revised: Jul 18, 2016 Accepted: Jul 29, 2016 Correspondence: Eun Soo Park Department of Plastic and Reconstructive Surgery, Soonchunhyang University Bucheon Hospital, 170, Jomaru-ro, Wonmi-gu, Bucheon 14584, Korea. E-mail: peunsoo@schmc.ac.kr

Copyright () 2016 The Korean Society for Aesthetic Plastic Surgery.

This is an Open Access article distributed under the terms of the Creative Commons Attribution Non-Commercial License (http://creativecommons.org/licenses/by-nc/4.0/) which permits unrestricted non-commercial use, distribution, and reproduction in any medium, provided the original work is properly cited. $\quad$ www.e-aaps.org
Here we present a case of a visible nodule on the infraorbital region resulting from foreign body granulomatous reaction to the injection of PLLA and its subsequent surgical management with a subciliary approach. We also discuss relevant injection techniques for minimizing the risk of subcutaneous nodule formation and treatment options for this complication.

\section{CASE REPORT}

A 46-year-old woman presented with an asymptomatic, visible, and palpable 5-mm nodule in the left infraorbital region (Fig. 1). Thirty-one months previously, she had undergone facial contouring with PLLA injections into the bilateral tear troughs and the cheeks for the correction of rhytides and age-associated volume loss. The patient was treated with 3 sessions of injections at 4-week intervals. Dilution of the product was conducted by adding $5 \mathrm{~mL}$ of bacteriostatic water and $1 \mathrm{~mL}$ of epinephrine-free lidocaine to each vial. It was reported that a depot injection technique at the level of the periosteum was used. The areas had been massaged deeply after injection with instructions given to the patient to repeat the massage. Three months after her last injection, visible nodule formation in the left infraorbital area was presented. Initially, she visited another physician, who began treatment with a 30-gauge needle followed by saline injection in an attempt to break up the nodule. Over the next 19 months, a series of 7 injections was performed, but it seemed to bring no improvement in the appearance of the nodule and the patient was referred to us. Ultrasound findings demonstrated a hypoechoic nodular lesion $(4 \times 8 \mathrm{~mm})$ at the subcutaneous level without abnormal vascularity (Fig. 2). 


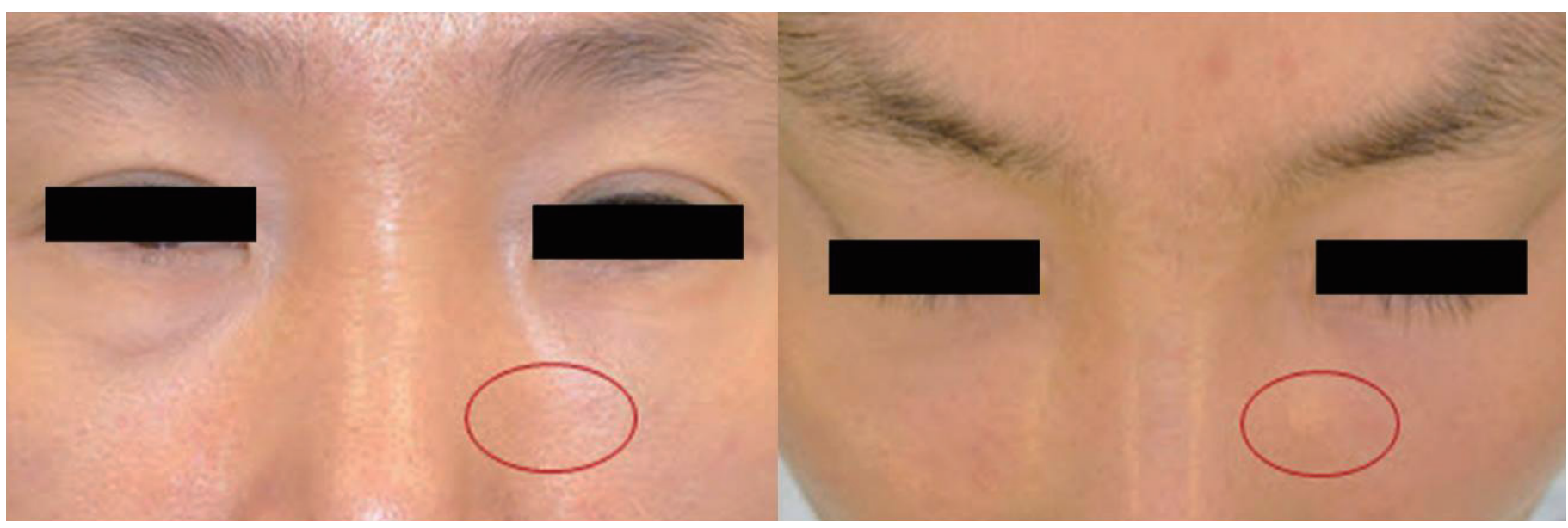

Fig. 1. Clinical photograph showing a subcutaneous nodule that is clearly visible and approximately $5 \mathrm{~mm}$ in size.

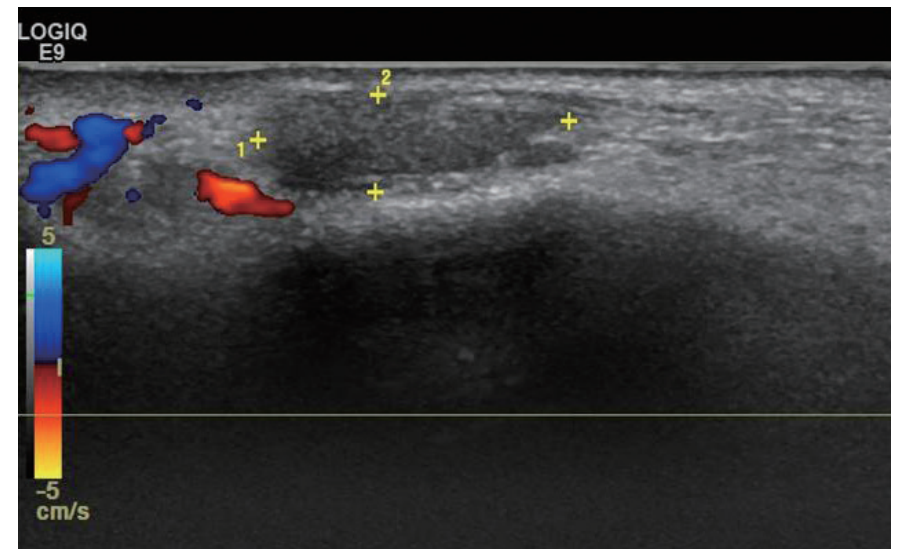

Fig. 2. Hypoechoic nodular lesion $(4 \times 8 \mathrm{~mm})$ at subcutaneous level without abnormal vascularity was revealed in ultrasound finding.

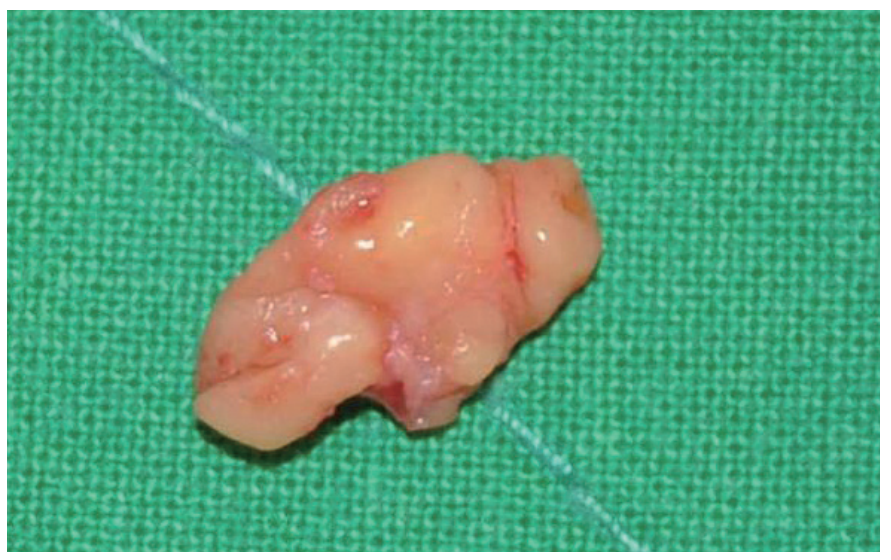

Fig. 3. Extracted granuloma appeared to be smooth and lobulated nodules.

Definite surgical excision was undertaken with a $10-\mathrm{mm}$ subciliary incision to remove the nodule. This nodule was extracted using dissecting scissors and forceps. On examination, the granuloma

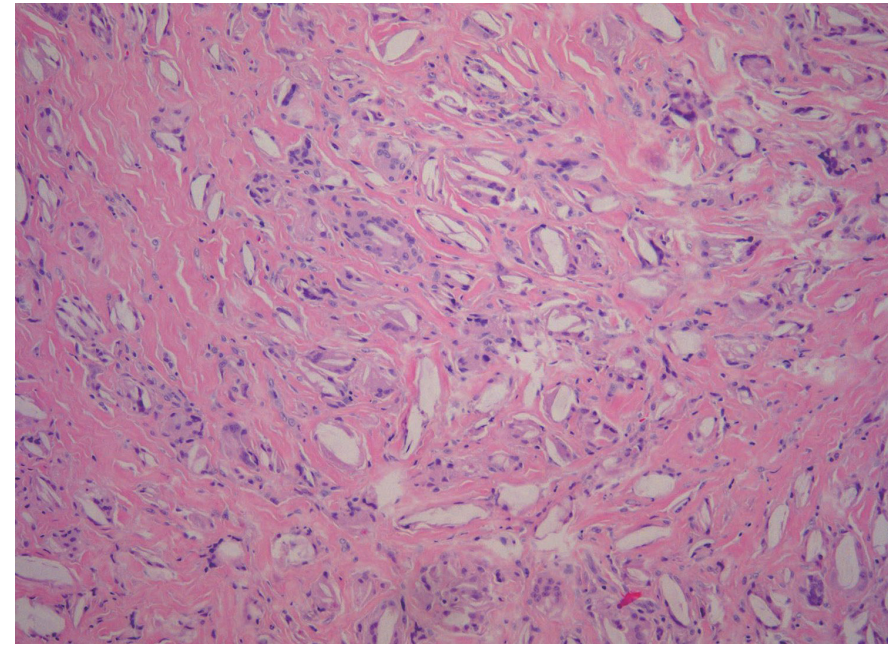

Fig. 4. At $\times 400$, histopathology of biopsied nodule demonstrates a foreign body granulomatous reaction pattern (hematoxylin and eosin [H\&E], $\times 100)$.

appeared to be composed of smooth and lobulated nodules (Fig. 3). Since it had been embedded in the orbicularis oculi muscle, the extracted nodule was larger than it had appeared clinically. Histopathologic examination revealed evidence of a foreign body granulomatous inflammatory reaction pattern consisting of abundant collagen formation around what appeared to be flakes of PLLA (Fig. 4). The incision was closed with 7-0 nylon and a sterile pressure dressing with ice pack was placed at the surgical site. The patient took antibiotics for 5 days. The wound healed well without complications (Fig. 5).

\section{DISCUSSION}

PLLA has gained attention for use as an effective soft-tissue filler for facial contouring with its ability to increase tissue volume and 


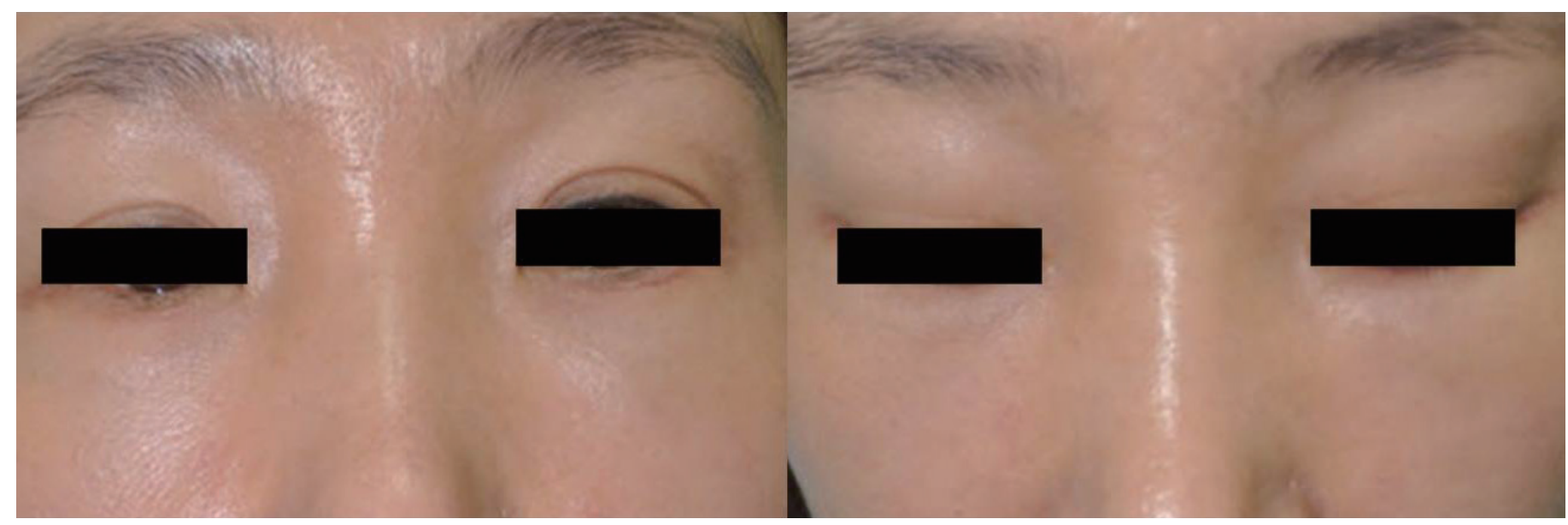

Fig. 5. The wound was healed well with minimal residual scarring 4 months after surgical excision.

dermal thickness, which improves the appearance of volume-loss aging through a minimally invasive approach. Following injection, the immediate effect is purely mechanical, created by the volume of Sterile Water for injection (SWFI). The SWFI is used to reconstitute PLLA; this is resorbed within 48 to 72 hours. Residual PLLA crystals are thought to elicit fibroblast production and endogenous collagen synthesis, resulting in gradual long-term improvement of aesthetic results [4].

Reviews of the data for this product have commonly concluded that it is safe and effective when injected into the skin of the face [5], while complications can include the development of subcutaneous nodules [6-8]. Although nodules are noted to typically be asymptomatic, nonvisible, and slightly palpable, they can be particularly disfiguring when these lesions occur in the relatively thin skin of the periorbital region compared with the buccal cheek area. This leads to long-term unacceptable aesthetic outcomes and therapeutic dilemmas. Therefore, use of injectable PLLA in the infraorbital area is currently not recommended [9]; however, if used in that area, it should be injected deeply under the orbicularis oculi muscle with postinjection massage. Furthermore, the smaller depot injection of PLLA and appropriate dilution of the product contribute to making this a safer approach.

The depth of injection plays a significant role in minimizing the occurrence of subcutaneous nodules. Injection should be directed into just above the level of the periosteum rather than the lower dermis $[10,11]$. Superficial injections increase the likelihood of a visible nodule. In addition to proper injection technique, proper dilution is critical when injecting PLLA, especially in the periorbital region. There have been reports in the literature that using dilutions of 5 to $10 \mathrm{~mL}$ may reduce side effects, which demonstrates an inverse relationship between volume dilution and the incidence of nodule formation $[2,11]$. Specifically, longer reconstitution times (24-72 hours prior to injection) and avoiding inclusion of precipitate from the wall of the PLLA vial in the syringe, may diminish the incidence of nodule formation. It is also believed that frequent massage of the treatment area after injection is helpful by more evenly dispersing PLLA particles [11,12]. Postinjection massage must be done in-office by a physician and continued by the patient.

As with any procedure, complications may occur. Multiple techniques are used to manage these nodules, including intralesional corticosteroid injections (triamcinolone acetonide, $3 \mathrm{mg} / \mathrm{mL}$ to 10 $\mathrm{mg} / \mathrm{mL}$ ) and breaking up the nodule with a needle after saline injection. Injection of hyaluronic acid filler is also used to make them less noticeable. However, results are variable, particularly in an area of thin skin such as the periorbital region. Recently, minimally invasive surgical slit excision for periorbital nodules has been advocated as a safe and effective method of treatment [13]. However, even proper execution including a small slit directly over the nodule as in the usual manner might leave a noticeable scar. With subciliary incision, the wound can be closed in a single layer and the scar will blend in the fine rhytides in the infraorbital region. This technique is minimally invasive and can leave minimal residual scarring.

Aesthetic management with PLLA can be complicated by disfiguring nodules, which may mask a granulomatous inflammatory reaction. Greater dilution, longer reconstitution times, lower injection dose per site, a deeper placement of the product, and frequent massage may yield decreased frequency of nodule formation. Use of this product in the periorbital region is best avoided, but if inescapable, PLLA should only be injected by experienced physicians who have trained appropriately. Patients receiving PLLA injections must be informed regarding its potential side effects.

In this case, after failing nonsurgical treatment options, the patient had excellent results with surgical excision of the persistent visible nodule that arose after PLLA injection in the infraorbital area. Based on our experience, for a patient with persistent visible nodule following PLLA injection, we suggest consideration of surgical intervention with a subciliary approach, which provides de- 
finitive removal of the nodule and minimal residual scarring. It is likely that clinical trials into this area will help to improve outcomes and provide more insight into treatment with this product.

\section{PATIENT CONSENT}

Patients provided written consent for the use of their images.

\section{REFERENCES}

1. Vleggaar D, Bauer U. Facial enhancement and the European experience with Sculptra (poly-l-lactic acid). J Drugs Dermatol 2004;3:5427.

2. Burgess CM, Quiroga RM. Assessment of the safety and efficacy of poly-L-lactic acid for the treatment of HIV-associated facial lipoatrophy. J Am Acad Dermatol 2005;52:233-9.

3. Vleggaar D. Soft-tissue augmentation and the role of poly-L-lactic acid. Plast Reconstr Surg 2006;118:46s-54s.

4. Gogolewski S, Jovanovic M, Perren SM, et al. Tissue response and in vivo degradation of selected polyhydroxyacids: polylactides (PLA), poly (3-hydroxybutyrate) (PHB), and poly (3-hydroxybutyrate-co3-hydroxyvalerate) (PHB/VA). J Biomed Mater Res 1993;27:1135-48.

5. Vleggaar D. Facial volumetric correction with injectable poly-L-lactic acid. Dermatol Surg 2005;31:1511-7; discussion 7-8.

6. Apikian M, Roberts S, Goodman GJ. Adverse reactions to polylactic acid injections in the periorbital area. J Cosmet Dermatol 2007;6:95101.

7. Hamilton DG, Gauthier N, Robertson BF. Late-onset, recurrent facial nodules associated with injection of poly-L-lactic acid. Dermatol Surg 2008;34:123-6; discussion 6.

8. Palm MD, Woodhall KE, Butterwick KJ, et al. Cosmetic use of poly-llactic acid: a retrospective study of 130 patients. Dermatol Surg 2010; 36:161-70.

9. Narins RS. Minimizing adverse events associated with poly-L-lactic acid injection. Dermatol Surg 2008;34 Suppl 1:S100-4.

10. Weinkle S. Minimizing the risk of adverse events with injectable polyL-lactic acid. Internet J Dermatol 2007;6:5668.

11. Lam SM, Azizzadeh B, Graivier M. Injectable poly-L-lactic acid (Sculptra): technical considerations in soft-tissue contouring. Plast Reconstr Surg 2006;118:55s-63s.

12. Vleggaar D. Poly-L-lactic acid: consultation on the injection techniques. J Eur Acad Dermatol Venereol 2006;20 Suppl 1:17-21.

13. Stewart DB, Morganroth GS, Mooney MA, et al. Management of visible granulomas following periorbital injection of poly-L-lactic Acid. Ophthal Plast Reconstr Surg 2007;23:298-301. 